\title{
Restauraciones estéticas de porcelana pura. Sistema Cercon
}

\section{Aesthetic all-ceramic restorations. Cercon System}

\author{
Fernández Bodereau E*, Bessone LM**, Cabanillas G***
}

\section{RESUMEN}

La estética en odontología está orientada a imitar la naturaleza, a tratar de conservar las proporciones, las formas, el color, la simetría. La tecnología ha desarrollado materiales cerámicos libres de metal que han reemplazado a los materiales tradicionales, capaces de imitar mejor a la naturaleza.

El óxido de circonio parcialmente estabilizado con itrio, no solo es extremadamente resistente, sino que posee la ventaja de ser altamente translucido. Su translucidez de aproximadamente el $50 \%$ de la luz incidente, permite la elaboración de restauraciones con apariencia natural. La duración del proceso de escaneado y fresado dependerá del tamaño del objeto, del número de objetos procesados y de los pasos seleccionados (CAM o CAD/CAM). La estructura de óxido de circonio densamente sinterizado con su preciso ajuste, se cubre con la cerámica de recubrimiento que ha sido especialmente desarrollada para este propósito. En casi todos los casos que se nos plantean hay un solo diagnóstico, pero distintas alternativas de plantear el tratamiento. El éxito de estas restauraciones será predecible siempre que se fundamente en el conocimiento de los principios biológicos que la sustentan. El objetivo de esta publicación es exponer de manera concisa las bases teóricas a modo esquemático y las imágenes de la secuencia del tratamiento clínico.

Palabras clave: Restauraciones estéticas, coronas de porcelana pura, prótesis de CAD-CAM, sistema Cercon.

\section{SUMMARY}

Aesthetic dentistry is dedicated to imitate nature by maintaining the size, shape, colour and symmetry. Technology has developed metal-free ceramic materials so capable of reproducing a natural appearance, that traditional materials have been replaced by them.

The yttrium partially-stabilized zirconium oxide does not only have the advantage of being extremelly resistant, but it is also highly translucent. It has a translucency that allows about $50 \%$ of the incident light to pass through, a characteristic that is vital for providing a more natural appearance to restorations. The duration of scanning and milling procedures will depend on the size of the object, the number of objects proccessed and the steps selected (CAM or CAD/CAM). The framework of the densely sintered zirconium oxide with its precise fit is veneered with the veneering ceramic developed especially for this purpose. Almost in all cases, there are several treatment alternatives for the same diagnosis.

The success of these restorations will be predicted as long as it is founded on sustaining biological principles. The aim of this paper is to concisely present the sequences for a clinical treatment in a schematic and illustrative manner.

Key words: Aesthetic resorations, all-ceramic crowns, CAD/CAM prosthesis, cercon system.

* Catedrático. Cátedra de Clínica de Prótesis Fija. Facultad de Odontología. Universidad Nacional de Córdoba. Argentina.

** Docente. Cátedra de Clínica de Prótesis Fija y Removible. Facultad de Odontología. Universidad Nacional de Córdoba. Argentina.

*** Profesor Adjunto. Cátedra de Clínica de Prótesis Fija y Removible. Facultad de Odontología. Universidad Nacional de Córdoba. Argentina. 
Fecha de recepción: 10 de enero de 2010.

Aceptado para publicación: 18 de marzo de 2010.

Fernández Bodereau E, Bessone LM, Cabanillas G. Restauraciones estéticas de porcelana pura. Sistema Cercon. Av. Odontoestomatol 2011; 27 (5): 231-240.

\section{INTRODUCCIÓN}

Los requisitos de cualquier restauración son que ésta sea resistente, duradera, precisa, funcional y estética. La porcelana pura ha sido desarrollada desde hace más de un siglo y presenta amplias posibilidades para la elaboración de restauraciones metal-free de máxima estética.

Las primeras restauraciones de porcelana pura surgieron en el año 1903, y contenían un alto porcentaje de feldespato (60\%), sílice (25\%) y fundentes (1, 2). Luego, en 1965, aparecieron las cerámicas reforzadas con alúmina $(3,4)$, donde la matriz vítrea de la porcelana es dispersada con cristales para aumentar la resistencia pero, en realidad, se compromete la estética por aumento de la opacidad. Por lo tanto para reconciliar los requerimientos estéticos y de resistencia se comenzó a utilizar una delgada cofia de alúmina, similar a un núcleo metálico, sobre el que se coloca la cerámica de recubrimiento (5).

Es importante destacar que el fortalecimiento de la porcelana se puede realizar a través de cuatro métodos:

- Refuerzo metálico (6)

- Refuerzo por dispersión de cristales cerámicos de alta resistencia y elasticidad en la matriz vítrea: cristales de óxido de aluminio, de óxido de leucita, y de óxido de mineral spinel de aluminio y magnesio, o bien

- Refuerzo cerámico por infusión de vidrio de baja fusión o

— Refuerzo por cristalización de vidrio (7).

Las restauraciones de porcelana pura en general presentan: óptima estética, conjugando opacidad con translucidez, su color es inalterable con el tiempo, presentan buena respuesta biológica, compatibilidad con los tejidos blandos en márgenes subgingivales, no sufren corrosión ni desgaste.
Algunos sistemas poseen Grababilidad lo que favorece su adhesión y, permiten una reducción vestibular más conservadora. La conductibilidad térmica de la cerámica es inferior al metal convirtiéndola en un elemento aislante y de protección al complejo dentino pulpar. Presentan algunos inconvenientes importantes como : su módulo de resistencia generalmente es inferior con respecto a una restauración metalocerámica, todos los sistemas exigen manipulación cuidadosa, y necesita cuidadosa preparación para otorgar soporte a la porcelana (8).

Se clasifican en:

- Restauraciones de cobertura total: coronas.

- Restauraciones de cobertura parcial:

a) Frentes laminares.

b) Incrustaciones inlays y onlays.

Las coronas de porcelana pura están indicadas en casos de $(4,9)$ :

1. Desgaste abrasivo de los dientes antagonistas.

2. Sector anterosuperior de la cavidad bucal con grandes demandas estéticas.

3. Cuando no se pueden restaurar por medios más conservadores.

4. Cuando el pilar aporta suficiente soporte.

5. Cuando se tiene respaldo de un laboratorio con experiencia en el sistema seleccionado.

Y sus contraindicaciones son:

- Cuando existe actividad parafuncional,

- Inadecuado soporte de la preparación dentaria,

- No se aconseja como pilar de puente, salvo en el sector anterior.

- En el sector anterior cuando hay sobremordida muy marcada.

Los sistemas cerámicos se clasifican según su método de producción: 
- Sistema por moldeado y sinterizado: Optec HSP/(Pentron-Jeneric), Duceram LFC (Degussa), In-ceram (Vivadent, Baldwin Park. Calif), Allceram (Formerly Cerestore Innotek Dental Corp).

- Sistema por inyección y presión: Empress (Ivoclar, Lichtenstein), IPS Empress 2 (Ivoclar, Lichtenstein), Optec OPC, SISTEMA IPS, E MAX, Ivoclar, Lichtenstein.

Su importancia radica en que: combina una alta resistencia flexural (400 Mpa) en dos niveles de opacidad, es decir mejora la estética. Por lo cual puede estar indicado para estructuras individuales anteriores y posteriores, estructuras de 3 unidades hasta el $2^{\circ}$ premolar como pilarsubestructuras de implantes (es decir coronas individuales y puentes de 3 unidades).

- Sistema por torneado o sustracción (CADCAM)

Cerec 3. Vita CEREC Mark II. (Vita Zahnfabrik) IDicor MGC. (Dentsply, L. D. Caulk División) Procera AllCeram. (Nobel Biocare, Goteborg, Suecia), Celay (Mikrona,Spreitenbach, Suiza), Los más utilizados en la actualidad son:

- SISTEMA CEREC 3 (Sirona), SISTEMA LAVA (3 M), SISTEMA PROCERA (Nobel Biocare),

- SISTEMA EVEREST (KAVO), etc.

\section{SISTEMA POR TORNEADO O SUSTRACCIÓN (CAD- CAM)}

Para la resolución de los casos clínicos que presentamos utilizamos el sistema Cercon (Degudent, Densply International Company) que describiremos.

\section{Sistema Cercon}

El sistema Cercon ha abierto nuevas vías de posibilidades de restauración para el clínico. Este sistema proporciona extraordinaria resistencia (entre 900 y $1.200 \mathrm{Mpa}$ ) y estética, de realizar con la capacidad puentes libre de metal con duración de largos períodos, así como coronas individuales. La adición de Cercon art (cerámica de recubrimiento) con su adecuado ajuste ha reducido algunas de las tensiones asociadas con la fabricación y las cuestiones de forma para el protésico dental, y el dentista se asegura de ofrecer un producto de estética con resultados previsibles de la restauración (10).

\section{Componentes:}

- Cercon eye: scaner óptico.

- Cercon brain: Unidad de fresado.

- Cercon heat: Horno de sinterizado.

Este sistema (11) se basa en el desarrollo realizado por investigadores suizos en el Instituto Federal de Tecnología de Zurich, en coordinación con la Escuela de Odontología de Zurich. El procedimiento fue descrito varias veces en la literatura con el nombre de DCM (direct ceramic machining). Finalmente, con la colaboración de Degudent fue optimizado y lanzado al mercado. El óxido de circonio o Y-TZP (12) (policristales de óxido de circonio tetragonales estabilizados con itrio), ha sido utilizado con mucho éxito desde 1969 en ortopedia, para cabezas de la articulación de la cadera artificiales. Este material ha sido utilizado predominantemente en odontología, para pernos radiculares prefabricados, brackets para ortodoncia o pilares transepiteliales de implantes.

En la actualidad se utiliza para:

- Coronas individuales anteriores o posteriores.

- Puentes con pilares terminales de hasta $47 \mathrm{~mm}$ de longitud anatómica en regiones anteriores y posteriores. El número de pónticos debe estar limitado a dos molares por tramo de puente.

- Puentes Inlay para la sustitución de dientes individuales con espesor máximo del póntico de $10 \mathrm{~mm}$.

Las contraindicaciones generales son el bruxismo y las parafunciones de resistencia al tratamiento.

El proceso del sistema Cercon (13) comienza con el escaneado óptico con láser del modelo en cera de la corona. Mediante un proceso informático, son fresados a partir de un bloque de Y-TZP presintetizado que posteriormente será sinterizado. Estos dos procesos, escaneado y corte tienen lugar en la unidad Cercon brain. El corte tiene dos fases, mientras se talla la parte áspera en un proceso inicial de prefresado, el segundo paso consiste en el ajuste preciso de la estructura. 


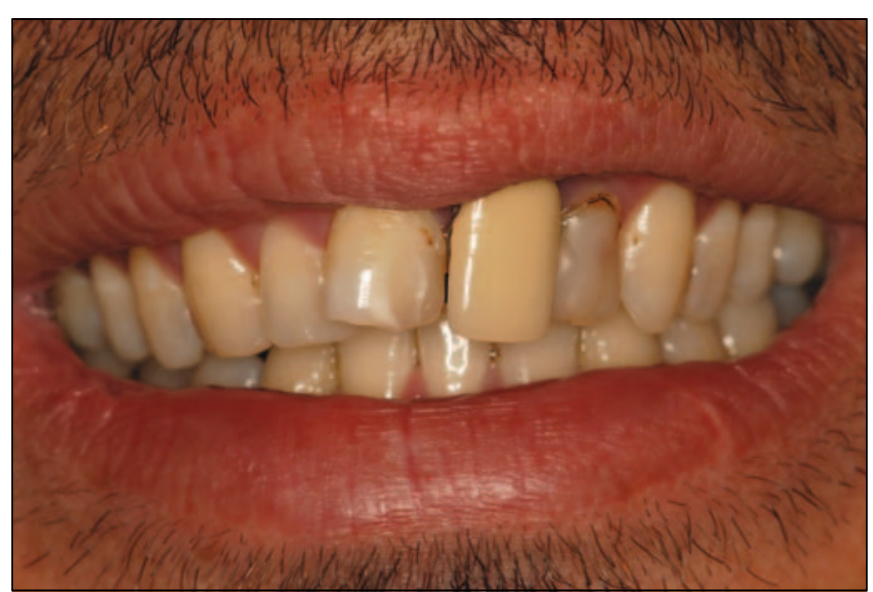

Fig. 1. El paciente se presentó con una restauración defectuosa por lo que se realiza radiografía para complementar el diagnóstico clínico. En la Rx obsérvese que el elemento 21 presentaba un poste deficiente con desadaptación, probable fractura de la raíz.

El objeto fresado se sinteriza en la unidad Cercon heat en un proceso térmico con una temperatura final de 1.350 grados centígrados. El proceso de sinterización completo lleva seis horas, dado que este proceso esta asociado con la contracción volumétrica del objeto fresado el corte sacado del bloque muestra una expansión de un 30\%. Esta expansión se duplica también en la unidad CERCON brain mediante un cálculo por ordenador tras el proceso de escaneado. Esto se hace posible porque el comportamiento de contracción de Cercon base, bloque de óxido de circonio puede determinarse de forma

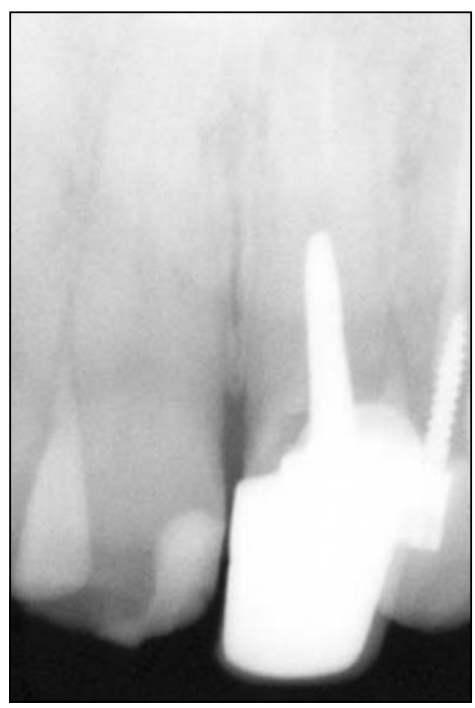

Fig. 2. En el examen clínico nótese la corona totalmente desadaptada en el elemento 21 , se decide realizar la extracción de este elemento. muy precisa durante la fabricación siendo por tanto muy predecible. La corona se completa con la cerámica de recubrimiento que corresponde (Cercon) que tenga el mismo coeficiente de expansión térmica que el núcleo para evitar las desadaptaciones a este nivel.

Presenta alta tolerancia frente a los tejidos gingivales, durabilidad máxima, acabado estético óptimo, no necesita exportación online, permite fresar circonio, cromo cobalto y poliamida pero de todos los materiales utilizados con el circonio se consigue el mejor acabado, tiempo de trabajo como la prótesis tradicional, y además el software se actualiza sin costo.

Sus inconvenientes son: No permite realizar puentes de más de 7 elementos, ni trabajos de prótesis híbridas sobre implantes.

\section{CASOS CLÍNICOS}

1. En el primer caso se realizó una prótesis parcial fija sobre implantes, logrando los objetivos planteados. Estética, función y predecibilidad de la restauración.

2. El segundo caso se desarrolló en un paciente con un implante en un segundo premolar superior, las expectativas del paciente eran lograr extremada estética por eso se recurre a la colocación de una corona de porcelana pura con

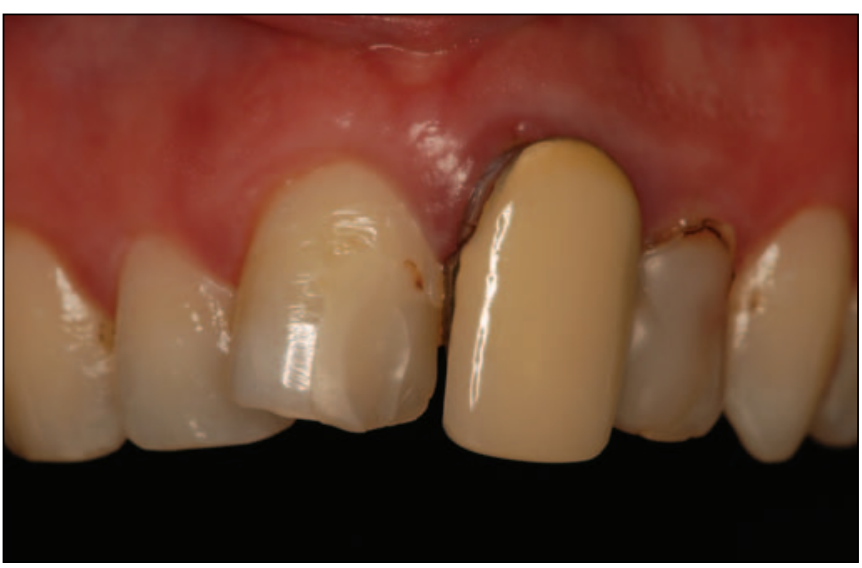

Fig. 3. Además los elementos 22,11 y 12 presentaban obturaciones estéticas deficientes. 
Fernández Bodereau E, Bessone LM, Cabanillas G. Restauraciones estéticas de porcelana pura. Sistema Cercon

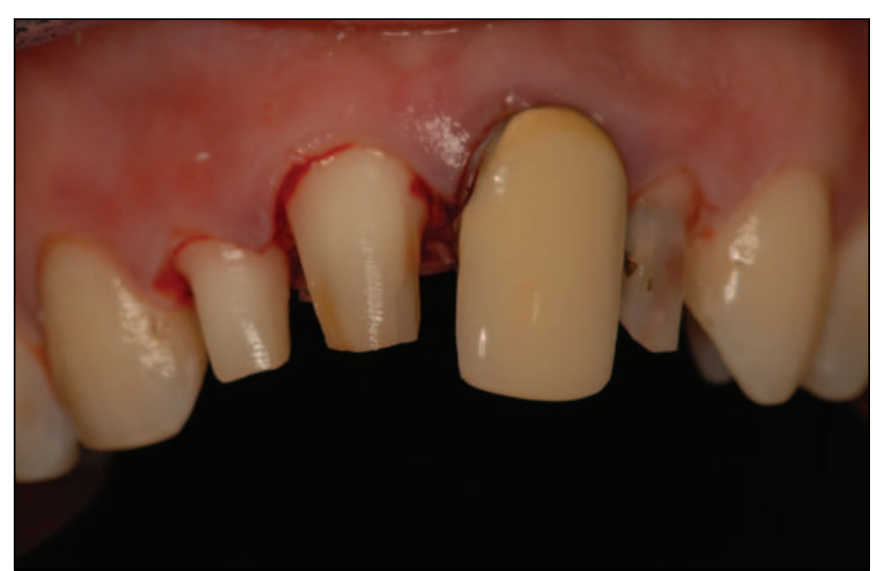

Fig. 4. El plan de tratamiento consistió en la elaboración de un puente de circonio con el sistema Cercon. Se tallaron primeramente los elementos pilares del puente.

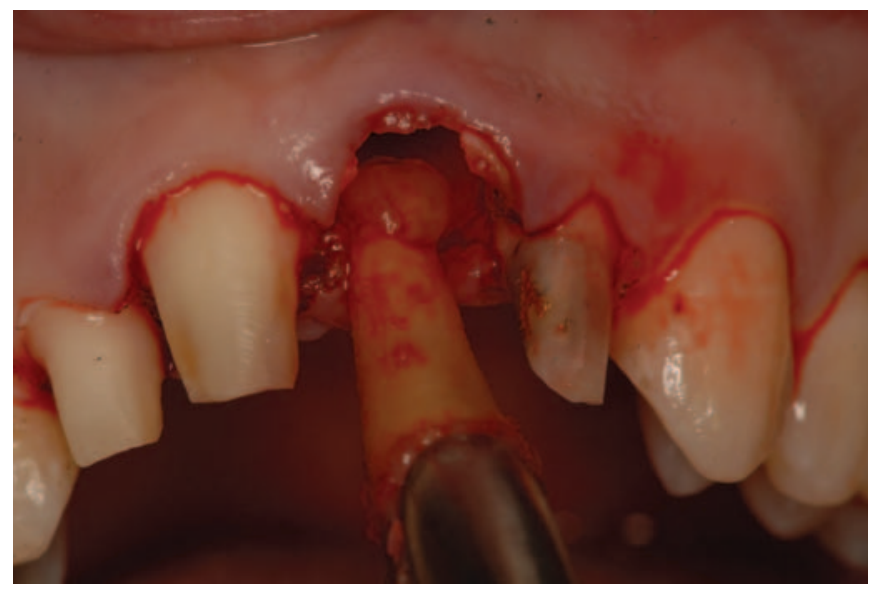

Fig. 5. Se realizó la exodoncia del elemento 21.

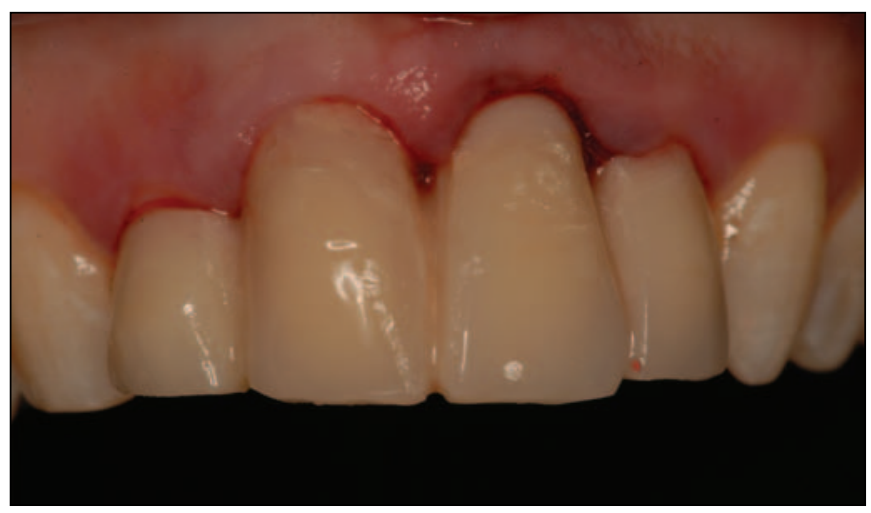

Fig. 6. Colocación de un provisorio con un póntico oval inmediato a la extracción, con la finalidad de conformar un perfil de emergencia adecuado, teniendo en cuenta no sólo la biología de los tejidos sino también las expectativas estéticas del paciente.

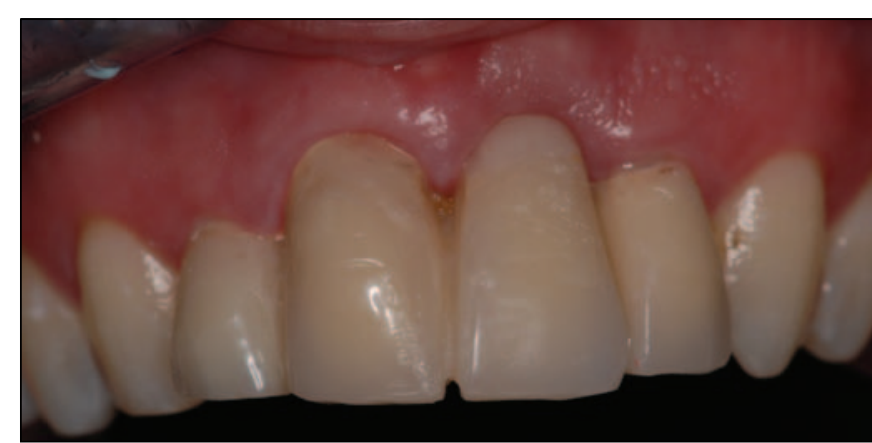

Fig. 7. Una semana después obsérvese la conformación y salud de los tejidos gingivales.

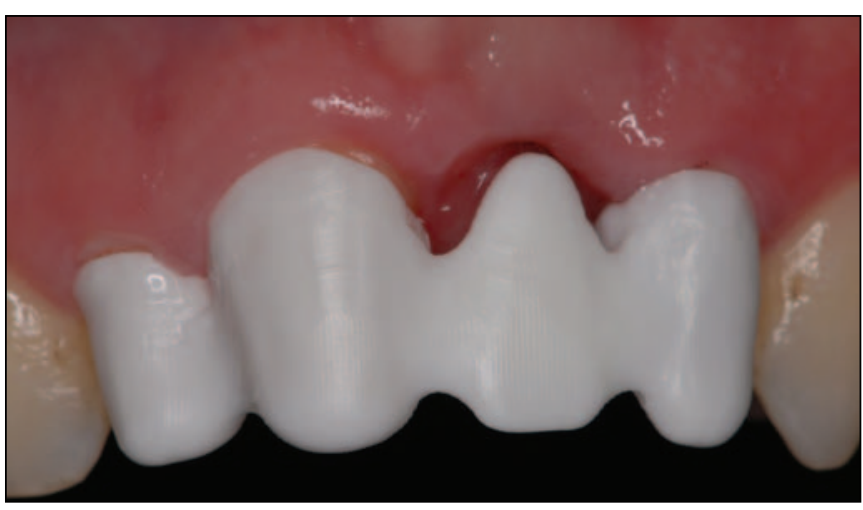

Fig. 8. Prueba clínica de la estructura de circonio. Obsérvese la adaptación de los márgenes.

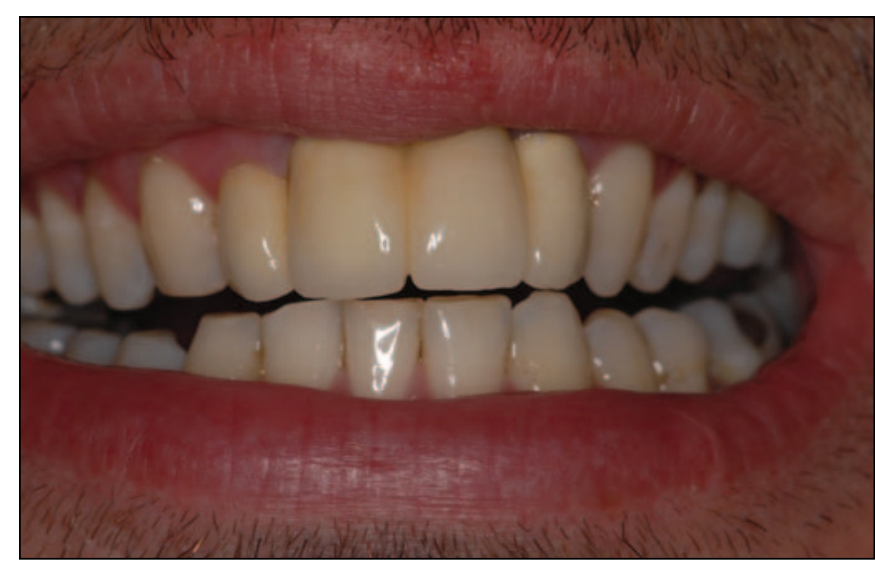

Fig. 9. El puente terminado y colocado en boca, nótese la estética lograda con la cerámica de revestimiento.

estructura de circonio y el empleo del sistema Cercon. La secuencia del tratamiento clínico se describen con las imágenes. 


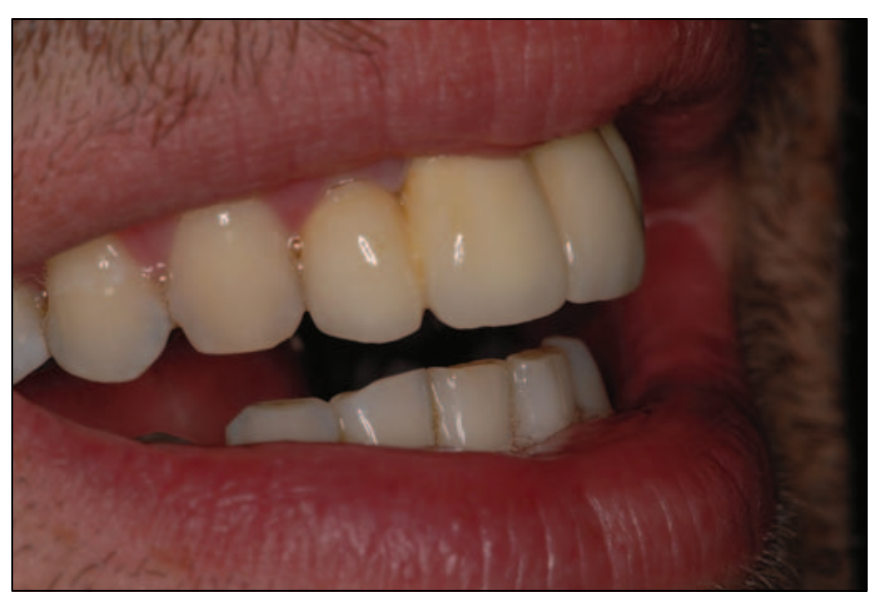

Fig. 10. Obsérvese el perfil de emergencia en una vista de costado, el estado de los tejidos periodontales, la armonía y la estética alcanzadas.

\section{DISCUSIÓN}

Existen tres factores importantes que fueron analizados en la literatura y que fundamentaron la utilización del sistema CERCON en los casos que presentamos: la estética, la resistencia a la fractura y la adaptación marginal.

En cuanto a la estética, numerosos autores realizaron investigaciones, mencionaremos sólo algunos de ellos, como Lou y col. (15), quienes evaluaron los resultados de este nuevo sistema en sus aspectos técnicos y clínicos. El sistema Cercon se aplicó clínicamente en coronas de cerámica y puentes en pacientes, todos los casos fueron seguidos en períodos de 1, 3, 6 y 12 meses después de la cementación de las restauraciones. El sistema Cercon demostró apariencia natural y estética. No hubo cambios de color, ni fracturas. Es decir que, la fiabilidad de todas las coronas de cerámica y puentes del sistema Cercon que se comprobó fue debido a los efectos de fortalecimiento de las cofias de circonio, y la indicación de este sistema puede extenderse a puentes posteriores con la posibilidad de lograr larga duración. Además, la mejor estética en comparación con otras técnicas de porcelana pura lo convierte en un sistema ideal para coronas y puentes de porcelana pura.

En contraposición, y respecto a la estabilidad del color y la previsibilidad del resultado estético se realizó un estudio (16) en el que se compararon 3 cerámicas de recubrimiento de los núcleos de circonio.

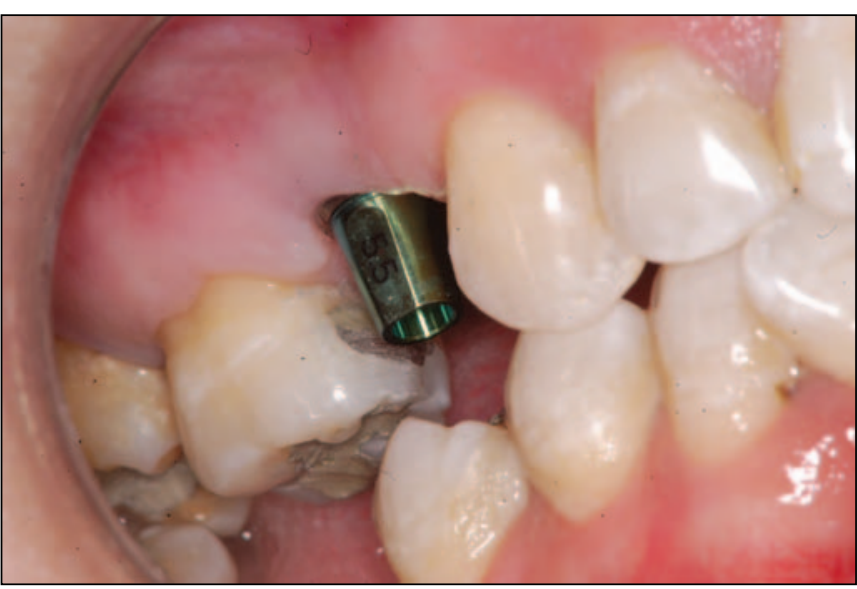

Fig. 11. En este caso, se realizó una corona de circonio sobre un implante BIOHORIZONS, simple solución, en un elemento premolar superior.

Las cerámicas de recubrimiento que se utilizaron fueron: cerámica A (inicial, GC), de cerámica B (Triceram, Esprident), y C, de cerámica (Cercon Ceram S, DeguDent). El color de las coronas y los dientes de referencia fue capturado mediante el análisis espectrofotométrico (SpectroShade, MHT), y la diferencia de color (deltae) se observaron mediante este método objetivo. Además, las coronas y los dientes de referencia se compararon subjetivamente por 11 observadores ciegos. Estos autores concluyeron que las 3 cerámicas cumplieron las exigencias estéticas sólo en una medida limitada. La cerámica B (Triceram, Esprident) para ellos permitió el resultado más previsible en términos de estabilidad de color, superando a cerámica C (Cercon Ceram).

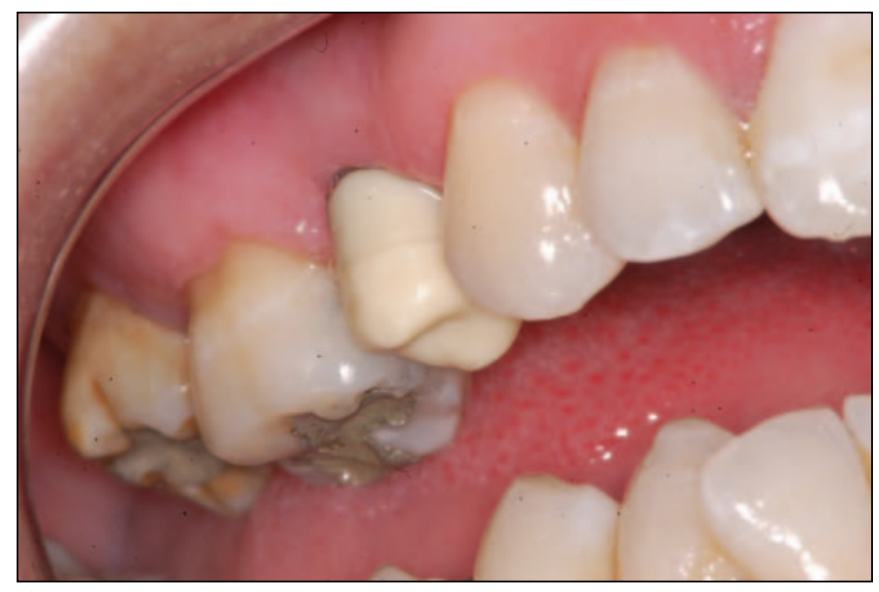

Fig. 12. Prueba clínica del núcleo de circonio. 


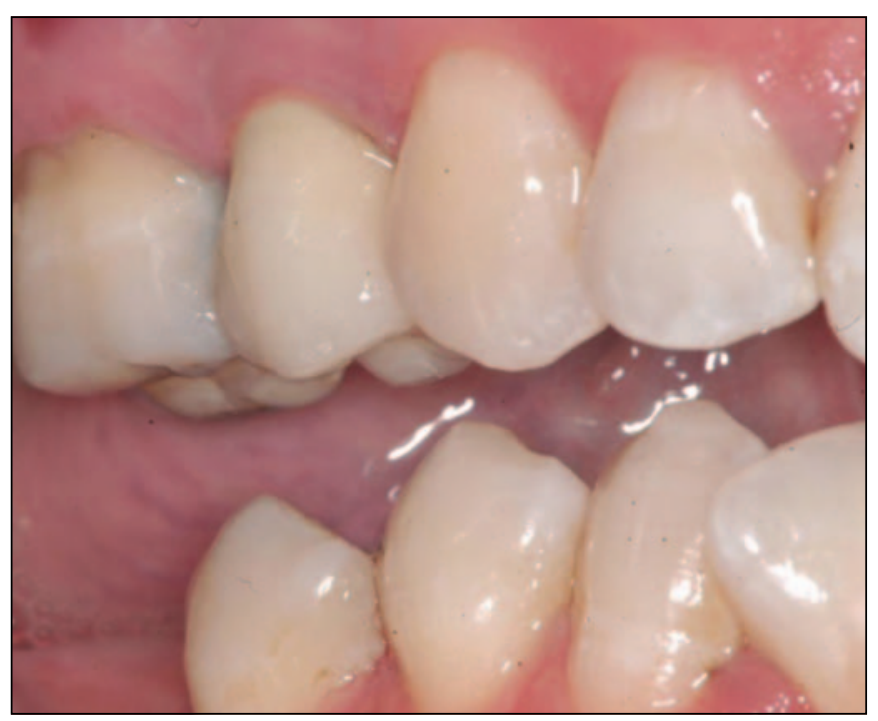

Fig. 13. Corona de circonio terminada. Nótese la estética y adaptación de los márgenes.

En cuanto a la adaptación marginal de las coronas, existen trabajos entre los que mencionaremos un ensayo clínico (17) donde se compararon coronas de cerámica vidrio fundido infiltrado Alumina/Zirconia y CAD/CAM Zirconia Utilizaron coronas INCERAM Zirconia y Cercon cementadas con un cemento de ionómero de vidrio. Utilizando un Sistema especial (CDA) para evaluar la calidad de las restauraciones en la línea de terminación marginal, la formación de placa y los resultados del índice gingival a 6 meses, 1 año y 2 citas de revisión al año fueron usadas para controlar los resultados periodontales de los tratamientos. Ningún signo clínico de decoloración marginal,

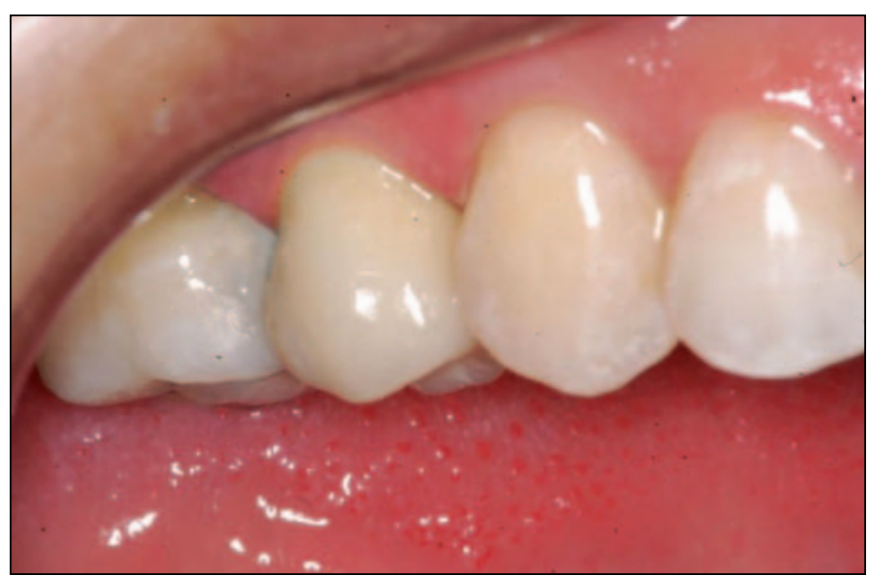

Fig. 14. A las dos semanas control de la restauración, obsérvese que los tejidos gingivales se presentan en perfecto estado. dolor persistente y caries secundaria fue detectado en ninguna de las restauraciones. De acuerdo con los criterios de CDA, la integridad marginal fue calificado como excelente para INCERAM Zirconia (73\%) y (80\%) restauraciones Cercon zirconia, respectivamente. La falta de coincidencia de color fue mayor para las restauraciones INCERAM Zirconia (66\%) que Cercon Zirconia (26\%). La placa y los resultados del índice gingival fueron en su mayoría cero y casi constante en el tiempo. Los cambios dependientes del tiempo en la placa y los resultados del índice gingival dentro y entre los grupos fueron estadísticamente similar $(p>0,05)$. Este estudio clínico demostró que las restauraciones unitarias INCERAM Zirconia y coronas Cercon son comparables en cuanto a los resultados clínicos, tanto como las modalidades de tratamiento.

La literatura clínica examinó a través de una revisión (18) la evidencia que apoya el uso de CAD/CAM y reconstrucciones con materiales reforzados con fibra. Se identificó las posibles pruebas a través de bases de datos (PubMed, EMBASE Medicamentos y Farmacología; Center for Reviews and Dissemination de la Universidad de York, la Biblioteca Cochrane, búsqueda manual de literatura no indexados, las búsquedas de referencia secundaria, y los contactos personales con ensayos clínicos de PI). Los Términos de búsqueda incluyeron: CAM restauraciones dentales de CAD/CAM, Cerec, Lava; Cercon; Procera, incrustaciones onlay, prótesis dentales, compuesto reforzado con fibra (FRC). Un total de 76 documentos fueron analizados por esta investigación. Concluyen que un gran número de estudios de cohortes independientes apoyan el uso de CAD/ CAM incrustaciones de cerámica/restauraciones onlay y coronas, pero existen complicaciones que inhiben la aplicación del examen. Excepto tal vez para postes endodónticos a base de fibra, la literatura FRC clínica no parece ser suficiente para la revisión de expertos. Además coinciden que los estudios in vitro actualmente no pueden servir como evidencia para la práctica clínica, excepto en casos limitados de simple función.

Otro estudio (19) del ajuste marginal de coronas de cobertura completa afirma que es un requisito importante para el éxito a largo plazo de este tipo de restauraciones. El propósito del estudio fue verificar la adaptación marginal de diseño asistido por ordenador (CAD)/equipo de fabricación asistida (CAM), las coronas de los dientes preparados. Cuatro tipos de 
materiales: cerámica de recubrimiento de zirconia- (DCZircon, DCS Dental, Allschwill, CH/S Cercon, Degussa, DeguDent GmbH, Hanau, Alemania), compuestos de recubrimiento reforzado con fibra (DC-Tell, DCS Dental/Gradia, GC Europa, Leuven, Bélgica), titanio-cerámica de recubrimiento (DC Titán, DCS Dental/Tikrom, Orotig, Verona, Italia) y compuestos de recubrimiento de titanio (DC Titán, DCS Dental/Gradia, GC Europa). Los valores de diferencia marginal se midieron en cuatro puntos (0 grados, 90 grados, 180 grados y 270 grados desde el centro de la superficie vestibular) en torno a la línea de terminación, en los dientes preparados. Las fotografías digitales fueron tomadas en cada punto de referencia y un programa informático fue utilizado para medir la cantidad de discrepancia marginal en micras. Los resultados mostraron que todos los materiales sometidos a prueba, con excepción de compuestos reforzados con fibra, muestran una adaptación marginal dentro de los límites de especificación de la ADA (25-40 micras). Dentro de las limitaciones de este estudio, se concluyó que el ajuste marginal de CAD/CAM es la restauración dentro de los límites considerados clínicamente aceptables por las especificaciones de la ADA \# 8.

Otras investigaciones (20) in vitro evaluaron el ajuste marginal de cuatro puentes de zirconia fabricado utilizando cuatro sistemas diferentes de fabricación asistida por ordenador (CAM). Tres sistemas (inLab, Everest, Cercon) son presinterizados y que tuvieron que ser sinterizadas a la densidad final después de la molienda, mientras que con un sistema (Digident) las restauraciones fueron directamente elaboradas con un material completamente sinterizado. Después de la fabricación, las discrepancias marginales horizontal y vertical, fueron determinados por medio de una técnica de réplica. Dentro de las limitaciones de este estudio, se podría concluir que el ajuste marginal depende significativamente del sistema CAD/CAM utilizado, con restauraciones elaboradas con circonio totalmente sinterizada se demostró un mejor ajuste de precisión.

Para evaluar la influencia de la configuración del núcleo de la restauración en la adaptación marginal en restauraciones anteriores unitarias. Komine y cols (21), utllizaron tres diferentes sistemas de CAD/CAM: Cercon Smart Cerámica (Grupo CE), Vita YZ/Cerec In-Lab (grupo YZ/CL), y Xawex (grupo XA). Fueron fabricados Núcleos de dos configuraciones diferentes (rectas y curvas) para cada grupo. La adaptación marginal de los mismos se midió en 60 puntos diferentes dentro del margen circunferencial completo usando un microscopio estereoscópico. Los valores de discrepancia marginal fueron comparados entre los dos diseños del núcleo y entre los tres grupos utilizando la prueba t-test. El nivel general de significación estadística fue de $5 \%$ después de corregir los valores de p utilizando el método de Bonferroni-Holm. Dentro de las limitaciones de este estudio, pudieron demostrar que la configuración marginal del núcleo influye en la adaptación marginal de las coronas que se fabrican con $\mathrm{ZrO}_{2}$ sinterizado parcialmente independiente del sistema CAD/CAM utilizado.

El presente estudio (22) evaluó la adaptación marginal e interna de cofias de cerámica de dióxido de circonio $\left(\mathrm{ZrO}_{2}\right)$ con coronas con tres diseños diferentes de línea de terminación. Se analizaron in vitro coronas de incisivos centrales superiores con los diseños de la siguiente línea final: el hombro recto (S), hombros redondeados (RS), y chaflán (C). Se realizaron cofias de cerámica $\mathrm{ZrO}_{2}$ fabricadas con un sistema CAD/CAM (Cercon Smart Cerámica), y las coronas se concluyeron por recubrimiento con una cerámica feldespática. Las medidas para la adaptación marginal e internas se realizaron en dos etapas. Se demostró que el diseño de línea determinación al parecer no ejercía influencia sobre la adaptación marginal de cofias de cerámica y coronas unitarias de $\mathrm{ZrO}_{2}$. Este estudio se contrapone en cierta medida a los anteriores.

El objetivo de otras investigaciones consistieron (22) en evaluar resistencia a la fractura a los impactos de los sistemas de cerámica y comprobar si la velocidad de carga afecta al mecanismo de la fractura. La energía absorbida por coronas (IPS) Empress y Cercon-Ceram $\mathrm{S}$ en una prueba de resistencia a la fractura fue comparado por la energía absorbida en una prueba de resistencia al impacto. Los principios de la fractografía se utilizaron para identificar el origen de la fractura y para calcular la tensión en el fracaso. Se utilizó Análisis de elementos finitos (FEA) para racionalizar los resultados. A pesar de la alta resistencia de los núcleos de circonio no hubo diferencia significativa en la energía absorbida entre los dos sistemas en el ensayo de resistencia al impacto. El modo dominante de fractura de todas las capas de restauraciones de cerámica bajo carga oclusal es 
un cono de grietas en la cerámica de recubrimiento. Se concluyó que la resistencia de la cerámica de recubrimiento tiene que mejorar, para explotar la alta resistencia de los núcleos de circonio.

Algunos autores estudiaron (23) el comportamiento a la fatiga de estrés de coronas con dos sistemas de cerámica con zirconia. Las dimensiones medias de la corona de un primer molar inferior, se importaron en el software de CAD, una preparación del diente fue modelada por la reducción de las paredes proximal de 1,5 mm y la superficie oclusal de 2,0 mm. La preparación del diente, basado en CAD se realizó con núcleos YTZP (sistema LAVA + cerámica LAVA 3M/ESPE, y recubrimiento de Vita + marco CERCON, Dentsply). Todas las pruebas mecánicas se realizaron por deslizamiento de un penetrador WC de 6,25 mm de diámetro 0,7 $\mathrm{mm}$ hacia lingual por el mesiodistal. Master curvas Weibull y la fiabilidad de las misiones de 50.000 ciclos de carga de 200 N fueron calculadas (Alta Pro 7, ReliaSoft). Las únicas cargas de fractura fueron a través del núcleo de circonio. La fiabilidad de una carga de $200 \mathrm{~N}$ x 50K ciclo no fue significativamente diferente entre los sistemas. LAVA y CERCON, mostraron comportamientos de fatiga similar; la carga de fatiga de los dos sistemas reproduce los modos de fallos similares.

El propósito de otros investigadores (24) fue evaluar y comparar las propiedades mecánicas de materiales de base de cerámica como la resistencia a la flexión biaxial y la fractura de las pruebas de resistencia. Las muestras fueron de materiales de cerámica (Finesse, Cergo, IPS Empress, In-Ceram Alumina, In-Ceram Zirconia, y Cercon Zirconia) fueron fabricados con un diámetro de $15 \mathrm{~mm}$ y una anchura de $1,2 \pm 0,2 \mathrm{~mm}$. Se encontraron diferencias significativas en la fuerza y los valores de dureza de los materiales evaluados. Cercon (material del núcleo de zirconia) mostró valores altos de resistencia a la flexión biaxial y resistencia a la fractura en comparación con las otras cerámicas estudiadas.

Rossenttrit y col (25) compararon la resistencia a la fractura in vitro de coronas elaboradas con sistemas de CAD/CAM y coronas de aleación de circonio. Se analizaron los siguientes materiales : una cerámica de alúmina deposición electroforética (Wolceram, Wolceram) y 4 de los sistemas basados en zirconia (Cercon, DeguDent; Digizon, Amann Girrbach y Lava,
3M ESPE) Se aplicaron 1.200 .000 cargas mecánicas de $50 \mathrm{~N} ; 3.000$ ciclos térmicos con agua destilada entre $5^{\circ} \mathrm{C}$ y $55^{\circ} \mathrm{C}$, (2 minutos por cada ciclo) para evaluar resistencia a la fractura, se determinaron los patrones de fractura y el tamaño de defecto. Se demostró que la fuerza de fractura varió entre 1.111 y $2.038 \mathrm{~N} \mathrm{~N}$ para la cementación convencional y entre 1.181 y 2.295 N N para los adhesivos. No se encontraron diferencias significativas entre el adhesivo y la cementación convencional. Los patrones de fractura se presentan principalmente como un astillamiento del recubrimiento. Se concluyó, además, que el material de la corona y la cementación no tienen ninguna influencia significativa en la fuerza de la fractura. Por lo tanto, para estos autores, los cementos adhesivos no son necesarios para la aplicación de la cerámica de alta resistencia.

\section{CONCLUSIONES}

- Los sistemas cerámicos de uso odontológico actualmente permiten la confección de restauraciones a través de variados métodos de producción como moldeado y sinterizado, inyección, presión, colado, y torneado (CAD/CAM), es decir, ya sea desde la sinterización sobre un muñón refractario hasta su elaboración con soporte informático.

- Su selección dependerá de múltiples factores, pero el más importante a nuestro entender, para evitar fallas en las restauraciones, es reunir el conocimiento necesario en el uso de los materiales y las técnicas basados en la evidencia científica.

- En nuestra experiencia, la adaptación marginal de estas restauraciones es en general muy exacta, más aún cuando se combinan con técnicas adhesivas de cementado que garantizan un cierre hermético del margen.

- El desarrollo de la cerámica está en pleno auge, superándose constantemente, e incorporando nuevas tecnologías en la confección de restauraciones cada vez más resistentes, precisas, y de simplificada fabricación y utilización.

\section{BIBLIOGRAFÍA}

1. Land Ch. A new system of restoring badly decayed teeth by means of an enameled metallic coating. Independent Pract 1886;7:407. 
2. Land Ch. Porcelain dental art. Dent Cosmos 1903;45:437-44.

3. Mc Lean JW,Hughes TH. The reinforcement of dental porcelain with ceramic oxide. Br Dent $\mathrm{J}$ 1965;119:251.

4. Mc Lean JW. The science and arto f dental ceramics monographs I, II (1974), III and IV (1976). Louisiana State Univ.

5. Chiche G,Pinalt A. Esthetics of anterior fixed prosthodontics. Quintessence Publishing Co., Inc. 1994.

6. Bruki CE, Ocampo RR. Compressive strengths of a new foil and porcelain fused-to-metalcrowns. J Prosthet Dent1987;57:404.

7. Castellani D. Differential treatment planning for the single anterior crown. Int $\mathrm{J}$ Periodont Rest Dent 1990;10:230.

8. Fradeani, M, Aquilano A. Clinical experience with Empress Crowns. Int $\mathrm{J}$ of Prosthodontics 1997; 10(3):241-7.

9. Shillingburg HT,Hobo S, Whitsett L. Fundamentos de Prostodoncia Fija. Quintessence Publishing Co., Inc 1981.

10. Poss S. CAD/CAM restorations: aesthetic all-ceramics, predictable fit. Dent today 2007;26(2): 86-8.

11. Filser F,Luthy $\mathrm{H}$,et al. All-ceramic dental Bridges by direct machining. Bioceram Proc In Symp Ceram Med 1997;10:371-6.

12. Luthardt R, Sandkuhl O, et al. Zirconia-TZP and alumina advanced Technologies for the manufacturing of single Clowns. Eur J Prosthodont Restor Dent 2000;7:113-9.

13. Mc Laren EA, White SN. Survival of inceram crowns in a private practice: a prospective clinical trial. J Prosthet Dent 2000;83:216-22.

14. Mc Laren EA, White SN. Glass infiltrated Zirconia/ alumina based ceramic for Clowns and fixed partialdentures. Pract Periodontics aesthet dent 1999;83:985-94.

15. Lou BY, Chao QY, Wang M, Lu Z, Chao YL. [Shortterm follow-up study of Cercon all-ceramic crowns and bridges][Article in Chinese] Hua Xi Kou Qiang Yi Xue Za Zhi 2004;22(5):402-3.

16. Sailer I, Holderegger C, Jung RE, Suter A, Thiévent B, Pietrobon N, Gebhard-Achilles W, Hämmerle $\mathrm{CH}$. Clinical study of the color stability of veneering ceramics for zirconia frameworks. Int J Prosthodont 2007;20(3):263-9.
17. Cehreli MC, Kökat AM, Akça K. CAD/CAM Zirconia vs. slip-cast glass-infiltrated Alumina/ Zirconia all-ceramic crowns: 2-year results of a randomized controlled clinical trial. J Appl Oral Sci 2009;17(1):49-55.

18. Kelly JR. Developing meaningful systematic review of $C A D / C A M$ reconstructions and fiberreinforced composites. Clin Oral Implants Res 2007;18 Suppl 3:205-17.

19. Romeo E, Iorio M, Storelli S, Camandona M, Abati S. Marginal adaptation of full-coverage CAD/CAM restorations: in vitro study using a nondestructive method. Minerva Stomatol 2009;58 (3):61-72.

20. Kohorst P, Brinkmann H, Li J, Borchers L, Stiesch M. Marginal accuracy of four-unit zirconia fixed dental prostheses fabricated using different computer-aided design/computer-aided manufacturing systems. Eur J Oral Sci 2009;117(3): 319-25.

21. Komine F, Iwai T, Kobayashi K, Matsumura H. Marginal and internal adaptation of zirconium dioxide ceramic copings and crowns with different finish line designs. Dent Mater J 2007;26(5):659-64.

22. Aboushelib MN, de Jager N, Kleverlaan CJ, Feilzer AJ. Effect of loading method on the fracture mechanics of two layered all-ceramic restorative systems. Dent Mater 2007;23(8):952-9.

23. Coelho PG, Silva NR, Bonfante EA, Guess PC, Rekow ED, Thompson VP. Fatigue testing of two porcelain-zirconia all-ceramic crown systems. Dent Materials 2009;25(9):1122-7.

24. Yilmaz H, Aydin C, Gul BE. Flexural strength and fracture toughness of dental core ceramics. J Prosthet Dent 2007;98(2):120-8.

25. Rosentritt $M$, Behr $M$, Thaller $C$, Rudolph $H$, Feilzer A. Fracture performance of computeraided manufactured zirconia and alloy crowns. Quintessence Int 2009;40(8):655-62.

\section{CORRESPONDENCIA}

Enrique Fernández Bodereau

Ituzaingó 1035

5000. Córdoba

Argentina

E-mail: bodereau@sinectis.com.ar 